\title{
Parameters Estimation of LFMCW Signals Based on Periodic Fractional Fourier Transform
}

\author{
Ying Liu ${ }^{1}$, Dianren Chen $^{1} \&$ Lei Chen ${ }^{1}$ \\ ${ }^{1}$ College of Electronic Information and Engineering, Changchun University of Science and Technology \\ Changchun, China
}

Correspondence: Lei Chen, Changchun University of Science and Technology, Changchun, China. Tel: 86-138-4304-7402. E-mail: chenlei511@126.com

$\begin{array}{lc}\text { Received: July 13, } 2014 & \text { Accepted: July 23, } 2014 \quad \text { Online Published: August 28, } 2014 \\ \text { doi:10.5539/mas.v8n5p143 } & \text { URL: http://dx.doi.org/10.5539/mas.v8n5p143 }\end{array}$

\begin{abstract}
When using fractional Fourier transform (FrFT) to detect and estimate linear frequency-modulated continuous wave radar signals, two problems will appear: multiple peaks occur in FRFT image and the output SNR at the true parameter values does not increase when the observation time is longer than the signals period. A multiple period LFMCW signals parameters estimation method based on period FRFT (PFRFT) is studied in this paper. The PFRFT formula of multiple period LFMCW signals is given. The relationship among PFRFT output SNR, observation time and sample signals SNR is analyzed. The estimation accuracy formula of PFRFT is derived. At last, numerical simulation shows the effectiveness of the algorithm and PFRFT is superior to FRFT for estimating a multiple periods LFMCW signals.
\end{abstract}

Keywords: LFMCW, FRFT, PFRFT, Parameter estimation, SNR

\section{Introduction}

Linear frequency-modulated continuous wave (LFMCW) signal is used in various fields, including radar, sonar and electronic monitoring. Frequency analysis method for the detection of non-stationary signals are commonly used,such as: Wigner-Ville distribution (WVD)、Short-time Fourier transform(STFT), fractional Fourier transform (FrFT) and so on[1]. WVD can be effectively used for a single period LFMCW signal parameter estimation, however, in the case of multi-periods signals, cross-term would interfere estimation of signals center frequency and modulation frequency; Smooth Pseudo Wigner-Ville Distribution1(SPWVD) and Time-frequency distribution series(TFDS) can effectively suppress the influence of cross terms while reduce the frequency resolution of the estimated parameters[2]. As the FrFT effectively overcomes the two problems above, the algorithm and related derivative algorithm have been widely studied and applied to the field of LFMCW signals detection and parameter estimation. P.R. White and others in-depth researched and analysis of the use of the FrFT performance parameters of chrip signals detection and estimation in the literature [3]. Qu Qiang and others studied the chirp signals detection and parameter estimation based on adaptive FrFT in the literature [4].C. Clemente and others use FrFT algorithm to study doppler and CS imaging algorithm. Liu Jiancheng and others detailed analyzed the fractional Fourier domain SNR of LFM signals under Gaussian white noise background in the literature [5].The Researches mentioned above do not give a method to improve the detection and parameter estimation performance of FrFT in the noise environment.

It is presented a method of estimation and detection LFMCW signals parameters based on PFRFT algorithm. When estimation and detection the parameters of a single period LFMCW signal, this algorithm and FRFT have the same advantages, but to estimate the parameters of the multi-periods LFMCW signals, this algorithm is better.

\section{PFrFT of LFMCW Signals}

\subsection{LFMCW Signals Mathematical Model}

The LFMCW signals shown in Figure 1, the mathematical expression of the m-th period of the LFMCW signals ${ }^{[7]}$ as follows:

$$
S_{m}(t)=A_{m} \cdot e^{j\left(2 \pi f_{m} t+\pi g_{m} \bmod \left(t, T_{z}\right)^{2}\right)}
$$


Where $A_{m}$ is the signals amplitude; $f_{m}$ is the signals initial frequency; $T_{z}$ is the period; $g_{m}$ is FM rate, $g_{m}=B_{m} / T_{z} ; B_{m}$ is the FM bandwidth; $j$ is the imaginary unit; $t$ is time variable, $\mathrm{m}=1 \ldots \mathrm{M}, \mathrm{M}$ is the number of the signals' period. The parameter of every LFMCWS signals' period is same. It is used the symbol $A, f$ and $g$ to express the amplitude, starting frequency and frequency modulation rate of the signals in this paper.

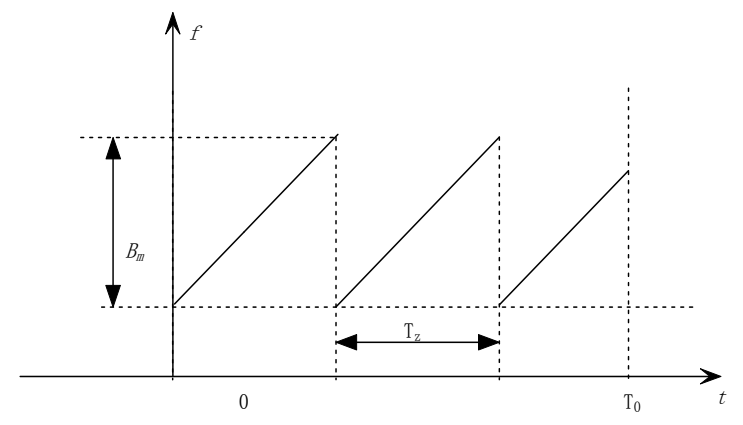

Fig. 1. LFMCW Signals

\subsection{FrFT}

There have a variety of definitions of FrFT due to the different application background. Although the physical interpretations of these definitions are not the same, they are equivalent in mathematics. One definition of arbitrary can be derived by other definitions. The FrFT of signal $s(t)$ can be expressed as follows ${ }^{[8]}$;

$$
\begin{gathered}
F_{R} K_{\alpha}(u, t)=\left\{\begin{array}{cc}
\sqrt{\frac{1-j \cot \alpha}{2 \pi}} e^{j \frac{t^{2}+u^{2}}{2} \cot \alpha-j u t \cdot \csc \alpha}, \alpha \neq n \pi \\
\delta(t-u), & \alpha=2 n \pi \\
\delta(t+u), & \alpha=(2 n+1) \pi
\end{array}\right.
\end{gathered}
$$

Where $\cot (\cdot)$ means the cotangent, $\operatorname{CSC}(\cdot)$ means the cosecant.

Two different situations due to the LFMCW signals' observation time is discussed as below:

(1)When the signals period number $\mathrm{M}=1$, observation time $\mathrm{T} \leq \mathrm{Tz}$, The signal can be expressed as $s_{1}(t)=A \cdot e^{j\left(2 \pi f \cdot t+\pi g \cdot t^{2}\right)}$, its FrFT can be expressed as:

$$
\begin{aligned}
\text { FRFT }_{s_{1}}(\alpha, u) & =\int_{-\infty}^{+\infty} K_{\alpha}(u, t) s_{1}(t) d t \\
& =\sqrt{\frac{1-j \cot \alpha}{2 \pi}} e^{j \frac{u^{2}}{2} \cot \alpha} \int_{0}^{T_{0}} A \cdot e^{j(2 \pi f-u \cdot \csc \alpha) \cdot t} \cdot e^{j\left(\pi g+\cdot \frac{\cot \alpha}{2}\right) \cdot t^{2}} d t
\end{aligned}
$$

$\operatorname{FRFT}_{s_{1}}(\alpha, u)$ achieves peak point at $2 \pi f-u_{0} \cdot \csc \alpha_{0}=0, \pi g+\frac{\cot \alpha_{0}}{2}=0$, then the estimation result is $f_{0}=\frac{u_{0} \cdot \csc \alpha_{0}}{2 \pi}, g_{0}=-\frac{\cot \alpha_{0}}{2 \pi}$.

(2)When the signals period number $\mathrm{M}>1$, observation time $\mathrm{T}=\mathrm{M} \times \mathrm{Tz}$, The signal can be expressed as:

$s_{2}(t)=A \cdot e^{j\left(2 \pi f t+\pi g \bmod \left(t, T_{z}\right)^{2}\right)}=\sum_{i=0}^{M-1} s_{1}\left(t+i \cdot T_{z}\right)$. Based on the Characteristics of $\mathrm{FrFT}^{[7]}$, the FrFT of the signal can be expressed as: 


$$
\begin{aligned}
F_{r F T_{s_{2}}}(\alpha, u)= & \int_{-\infty}^{+\infty} K_{\alpha}(u, t) s_{3}(t) d t=\int_{-\infty}^{+\infty} K_{\alpha}(u, t) \sum_{i=0}^{M-1} s_{1}\left(t-i \cdot T_{z}\right) d t \\
= & \sum_{i=0}^{M-1} \int_{-\infty}^{+\infty} K_{\alpha}(u, t) s_{1}\left(t-i \cdot T_{z}\right) d t \\
= & \sum_{i=0}^{M-1} F R F T_{s_{1}}\left(\alpha, u-i \cdot T_{z} \cos \alpha\right) e^{j\left(\frac{\left(i \cdot T_{z}\right)^{2}}{2} \sin \alpha \cos \alpha-u \cdot i \cdot T_{z} \cdot \sin \alpha\right)} \\
= & \sum_{i=0}^{M-1} \sqrt{\frac{1-j \cot \alpha}{2 \pi}} e^{j \frac{\left(i \cdot T_{z}\right)^{2}}{2} \cot \alpha} \cdot e^{j\left(\frac{\left(i \cdot T_{z}\right)^{2}}{2} \sin \alpha \cos \alpha-u \cdot i \cdot T_{z} \cdot \sin \alpha\right)} \\
& \cdot \int_{0}^{T_{0}} A \cdot e^{j\left(2 \pi f-\left(u-i \cdot T_{z} \cos \alpha\right) \cdot \csc \alpha\right) \cdot t} \cdot e^{j\left(\pi g \cdot+\cdot \frac{\cot \alpha}{2}\right) \cdot t^{2}} d t
\end{aligned}
$$

By formula (5), it can be seen that the $\operatorname{FrFT}_{s_{2}}(\alpha, u)$ achieves peak points at $2 \pi f-\left(u-i \cdot T_{z} \cos \alpha\right) \cdot \csc \alpha=0$ 、 $\pi g+\frac{\cot \alpha_{0}}{2}=0$.so $u=\frac{2 \pi f_{0} u_{0}}{\csc \alpha_{0}}+i T_{z} \cdot \cos \alpha, \alpha_{0}=-\cot ^{-1} 2 \pi g_{0}$. In this case the FM rate estimation is correct. Due to $\mathrm{i}=0,1 \ldots \mathrm{M}-1$, u has 5 values, FRFT results will appear $\mathrm{M}$ peaks, so the starting frequency can not be accurately estimated.

Using MATLAB to simulate the above situation, the radar echo parameters are shown in the following table.The simulation results shown in figure (2):

Table 1. Simulation parameters

\begin{tabular}{lll}
\hline \multicolumn{1}{c}{ Signals parameters } & \multicolumn{1}{c}{ (1) } & \multicolumn{1}{c}{ (2) } \\
\hline Start frequency & $30 \mathrm{~Hz}$ & $30 \mathrm{~Hz}$ \\
Frequency modulation rate & $8 \mathrm{~Hz} / \mathrm{s}$ & $8 \mathrm{~Hz} / \mathrm{s}$ \\
period & $2.56 \mathrm{~s}$ & $2.56 \mathrm{~s}$ \\
The sampling frequency & $200 \mathrm{~Hz}$ & $200 \mathrm{~Hz}$ \\
Number of cycles & 1 & 3 \\
Signals to noise ratio & $-5 \mathrm{~dB}$ & $-5 \mathrm{~dB}$ \\
\hline
\end{tabular}

In figure (2), (a) (b) (c) means the projection on $\alpha$-axis, $\mu$-axis and two-dimensional map of signals FRFT when $M=1$. By calculation, the results of simulation and practical results are the same.(d) (e) (f) means the projection on $\alpha$-axis, $\mu$-axis and two-dimensional map of signals FRFT when $M=3$. Graph (a) (d) peak coordinates are consistent, that is to say, signals modulation frequency estimation is correct, Fig. (e) (f) have 3 peak values respectively, that will interfere estimation of initial frequency. At the same time, comparing $M=1$ and $M=3$, the output signals to noise ratio does not significantly improve while increasing the sample signals observation time. It is considered that FRFT not optimal multi-periods LFMCW signals parameter estimation algorithm.
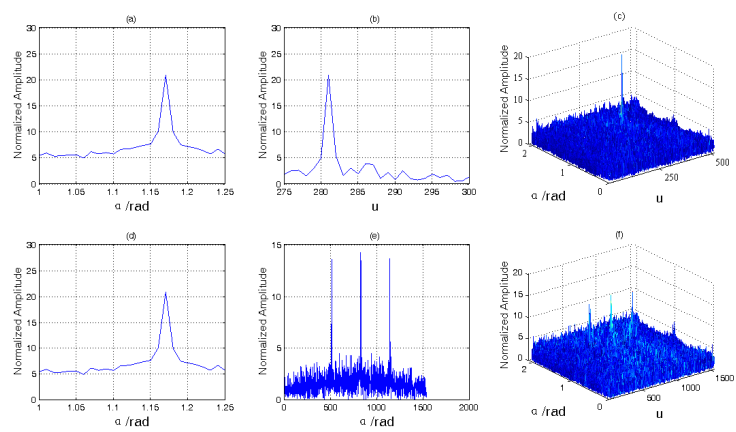

Figure 2. The sample signals FRFT when $M=1$ and $M=3$ 


\subsection{FRFT output SNR}

Since the FrFT on the $\alpha$ shaft $(0,2 \pi)$ symmetry ${ }^{[8]}$, in order to facilitate analysis, we take $0<\alpha<\pi / 2$.Assuming Gaussian white noise conditions, the observe signals is $x(t)=s(t)+n(t), 0<t<T_{0}$, $T_{0}$ means signals observation time, $n(t)$ is Gaussian white noise whose mean is 0 and variance is $\sigma^{2}$. Literature [5] proposed that the square of the peak on two-dimensional FrFT domain is signals power, and the square of the peak of the noise as the noise power. The FRFT output SNR of the LFMCW signals can be expressed as ${ }^{[8]}$ :

$$
\operatorname{SNR}_{F R F T}\left(u_{0}, \alpha_{0}\right)=\frac{A^{2} T_{0}^{2}}{2 A^{2} T_{0} \sigma^{2}+\sigma^{4}}=\frac{S N R_{\text {in }}^{2} \cdot T_{0}^{2}}{2 T_{0} \cdot S N R_{\text {in }}+1}
$$

Where ${ }^{[0]}: \quad S N R_{i n}=A^{2} / \sigma^{2}$.

Combining formula (6) and figure 2, simulated the FRFT output SNR with the parameters in Table 1, the result is shown in Figure 3.The results shows that when the observation time $\mathrm{T}_{0}>\mathrm{T}_{\mathrm{z}}$, the SNR in the peak does not increase, while $\mathrm{T}_{0}<\mathrm{T}_{\mathrm{z}}$, the output SNR increases with the observation time increase. It is considered that FRFT is not optimal parameter estimation algorithm when the numbers of the signals' period $\mathrm{M}>1$.

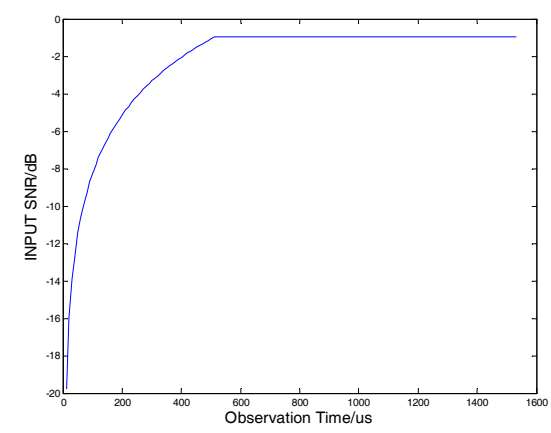

Figure 3. Relationship between FRFT output SNR and observation time

\subsection{Periodic FrFT}

Through the above analysis, it finds that LFMCW signals estimate by traditional FRFT, when M=1, FRFT can effectively estimate the parameters, and the SNR in the peak increases with the observation time increasing. When M>1, FRFT result appears $M$ peaks that interfere starting frequency estimation, although it could effectively estimate the FM rate of the signals, and when the observation time is longer than Tz, the SNR no longer increase, the max value is $\frac{S N R_{i n}^{2} \cdot T_{z}^{2}}{2 T_{z} \cdot S N R_{i n}+1}$. That means FRFT is not optimal during multiple periods of LFMCW signals parameter estimation. Therefore this paper presents a Multi-cycle LFMCW parameter estimation method base on period FRFT algorithm, definitions LFMCW signals parameter field $\Omega=\left(f, g, \widetilde{T}_{z}\right)$, where $\mathrm{f}, \mathrm{g}, \widetilde{T}_{z}$ means the starting frequency, modulation frequency and period respectively, when $\mathrm{f}, \mathrm{g}$ is in the parameter estimation results use $\alpha, u$ representing, signals parameter field $\Omega=\left(f, g, \widetilde{T}_{z}\right)$, definition of period FrFT is as follows:

$$
\operatorname{PFRFT}_{s}\left(\Omega^{\prime}\right)=\int_{-\infty}^{+\infty} F_{\alpha}\left(u, t, \widetilde{T}_{z}\right) s(t) d t
$$

Where 


$$
F_{\alpha}\left(u, t, \widetilde{T}_{z}\right)=\left\{\begin{array}{cc}
\sqrt{\frac{1-j \cot \alpha}{2 \pi} e^{j \frac{\left.\left(\bmod ,, \widetilde{T}_{z}\right)\right)^{2}+u^{2}}{2} \cot \alpha-j u t \cdot \csc \alpha}}, \alpha \neq n \pi \\
\delta(t-u), & \alpha=2 n \pi \\
\delta(t+u), & \alpha=(2 n+1) \pi
\end{array}\right.
$$

Where $\bmod (\bullet)$ means modulo operation, with formula (1) obtains

$$
\begin{aligned}
\operatorname{PFRFT}_{s_{1}}\left(\Omega^{\prime}\right) & =\int_{-\infty}^{+\infty} F_{\alpha}\left(u, t, \widetilde{T}_{z}\right) s_{1}(t) d t \\
& =\sqrt{\frac{1-j \cot \alpha}{2 \pi}} e^{j \frac{u^{2}}{2} \cot \alpha} \int_{0}^{T_{0}} A \cdot e^{j(2 \pi f-u \cdot \csc \alpha) \cdot t} \cdot e^{j \pi g \cdot \bmod \left(t, T_{z}\right)^{2}+\frac{\cot \alpha}{2} \bmod (t, \widetilde{T})^{2}} d t
\end{aligned}
$$

The physical meaning of the formula (9), searches the ture period with the multi-period LFMCW signals and do FrFT after fold-weighted with the ture period, then search the peak value in the three-dimensional parameter field and get the coordinates of the peak, at last, calculate the exact value of the signals' parameters.
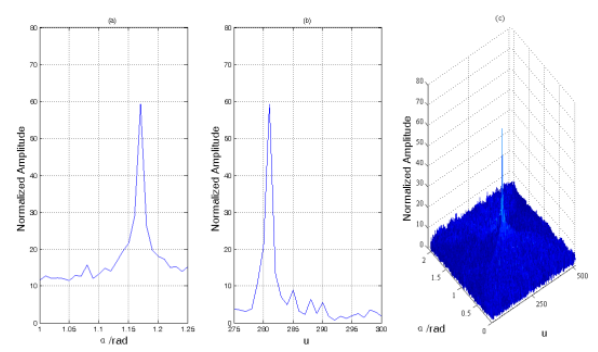

Figure 4. M=3 LFMCW PFRFT results

To simulate the performance of PFrFT with the parameters in Table 1, the result as shown in Figure 4. in the case of $\mathrm{M}=3$, there is a peak in the two-dimensional domain consisting of $\alpha, u$, PFRFT output of $f$, $g$ parameter estimates are correct by calculation, and the peak amplitude is 60 which 3 times of the peak amplitude in figure 2 ,and SNR in the peaks is significantly higher .

Figure 5 shows the value curve of the peak when searching the value of period $\widetilde{T}_{z}$. Maximum peak is achieved in the period $\widetilde{T}_{z}=2.56 \mathrm{~s}$, it is consistent with the actual value

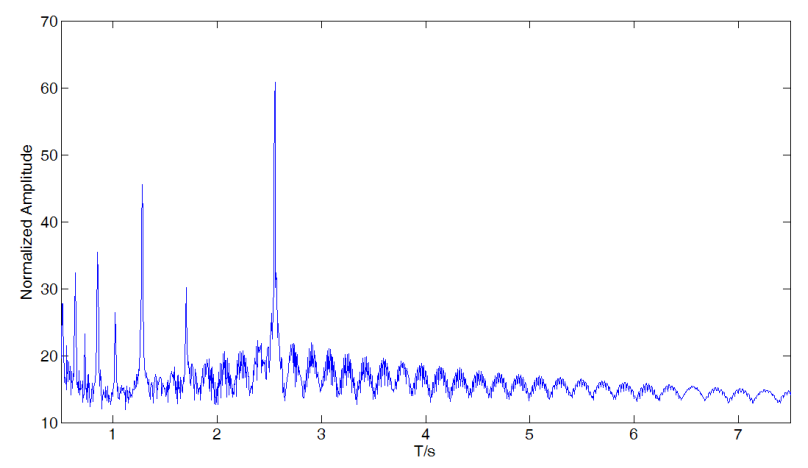

Figure 5. PFRFT output amplitude of the peak point in different period $\mathrm{Tz}$ 


\section{PFRFT Performance Analysis}

When analyzing PFRFT output SNR, it lets $\left|\operatorname{PFRFT}_{x}(\Omega)\right|^{2}$ as observing statistic. Assuming Gaussian white noise environment, the observed signals is $x(t)=s(t)+n(t) \quad t \leq T_{0}$ SNR is $S N R_{i n}$, observation time is expressed as $T_{0}, n(t)$ is Gaussian white noise whose mean is 0 'and variance is $\sigma_{0}^{2}$. Defineds:

$$
\begin{aligned}
& P_{x}(\Omega)=\left|\operatorname{PFRFT}_{x}(\Omega)\right|^{2}=\left|\int_{-\infty}^{+\infty} \int_{-\infty}^{+\infty} F_{\alpha}(\Omega) s_{1}\left(t_{1}\right) F_{\alpha}{ }^{*}(\Omega) s_{1}{ }^{*}\left(t_{2}\right) d t_{1} d t_{2}\right| \\
& =\left|\operatorname{PFRFT}_{s}(\Omega)\right|^{2}+\left|\operatorname{PFRFT}_{n}(\Omega)\right|^{2}+2 \operatorname{Re}\left[\operatorname{PFRFT}_{s}(\Omega) \cdot \operatorname{PFRFT}_{n}{ }^{*}(\Omega)\right]
\end{aligned}
$$

Where $\operatorname{Re}(\cdot)$ is realistic calculation, * is conjugate, $n(t)$ is Gaussian white noise, so the above equation can be written as:

$$
\begin{aligned}
& P_{x}(\Omega)=\left|\operatorname{PFRFT}_{x}(\Omega)\right|^{2} \\
& =\left|\operatorname{PFRFT}_{s}(\Omega)\right|^{2}+2 \operatorname{Re}\left[\operatorname{PFRFT}_{s}(\Omega) \cdot \operatorname{PFRFT}_{n}^{*}(\Omega)\right]
\end{aligned}
$$

\subsection{Output $S N R$}

According to Redefinition of SNR in the literature[5], PFrFT output SNR can be expressed as:

$$
S N R_{\text {PFRFT }}=\frac{\left|\operatorname{PFRFT}_{s}\left(\Omega_{0}^{\prime}\right)\right|^{4}}{\operatorname{var}\left(P_{x}\left(\Omega_{0}^{\prime}\right)\right)}
$$

Where $\Omega_{0}^{\prime}$ is the parameter of peak, $\operatorname{var}(\bullet)$ is variance calculation.

According to formula (7), $\left|\operatorname{PFRFT}_{x}(\Omega)\right|^{2}$ at the peak of PFRFT could be expressed as:

According to formula (13):

$$
\left|\operatorname{PFRFT}_{s}\left(\Omega_{0}^{\prime}\right)\right|^{2}=A^{2} T_{0}^{2} \frac{\csc \alpha_{0}}{2 \pi}
$$

$$
\begin{gathered}
E\left(P_{x}\left(\Omega_{0}^{\prime}\right)\right)=\mathrm{E}\left(\left|\operatorname{PFRFT}_{s}(\Omega)\right|^{2}+2 \operatorname{Re}\left[\operatorname{PFRFT}_{s}(\Omega) \cdot \operatorname{PFRFT}_{n}^{*}(\Omega)\right]\right) \\
=A^{2} T_{0}^{2} \frac{\csc \alpha_{0}}{2 \pi}+\frac{T_{0} \sigma_{n}^{2} \csc \alpha}{2 \pi} \\
E\left(\left|P_{x}\left(\Omega_{0}^{\prime}\right)\right|^{2}\right)=E\left(\left|\operatorname{PFRFT}_{s}(\Omega)\right|^{4}+4 \operatorname{Re}\left[\operatorname{PFRFT}_{s}(\Omega) \cdot \operatorname{PFRFT}_{n}(\Omega)\right] \cdot\left|\operatorname{PFRFT}_{s}(\Omega)\right|^{2}\right. \\
\left.\left.+4 \operatorname{Re}^{2}\left[\operatorname{PFRFT}_{s}(\Omega) \cdot \operatorname{PFRT}_{n}(\Omega)\right]\right)\right)
\end{gathered}
$$

Then

$$
\begin{aligned}
& \operatorname{var}\left(\left|\operatorname{PFRFT}_{s}\left(\Omega_{0}^{\prime}\right)\right|^{2}\right)=E\left(\left|\operatorname{PFRFT}_{x}\left(\Omega_{0}^{\prime}\right)\right|^{4}\right)-E^{2}\left(\left|\operatorname{PFRFT}_{s}\left(\Omega_{0}^{\prime}\right)\right|^{2}\right) \\
& =\frac{T_{0}^{2}\left(1+\csc ^{2} \alpha_{0}\right)}{4 \pi^{2}}\left[2 A^{2} T_{0} \sigma_{n}^{2}+\sigma_{n}^{4}\right]
\end{aligned}
$$

So

$$
S N R_{P F R F T}=\frac{\left(A^{2} T_{0}{ }^{2} \frac{\csc \alpha_{0}}{2 \pi}\right)^{2}}{\frac{T_{0}^{2}\left(1+\csc ^{2} \alpha_{0}\right)}{4 \pi^{2}}\left[2 A^{2} T_{0} \sigma_{n}^{2}+\sigma_{n}^{4}\right]}=\frac{S N R_{i n}^{2} \cdot T_{0}^{2}}{2 T_{0} \cdot S N R_{i n}+1}
$$

Comparing the formula (17) and formula (6), PFrFT and FrFT of LFMCW signals have the same output SNR expression. But the value range of $T_{0}$ is not the same, $T_{0}$ in the formula (6) is not more than $T_{z}$ and $T_{0}$ in the formula (17) means sample observation time. It shows that PFrFT and FrFT of the signals have the same output SNR when the sample observation time is less than or equal the period of the signal. But with increasing sample observation time, FrFT output SNR does not increase, while PFrFT output SNR continues increasing, PFrFT takes full advantage of all the information of sample signals. As shown in Figure (6). 


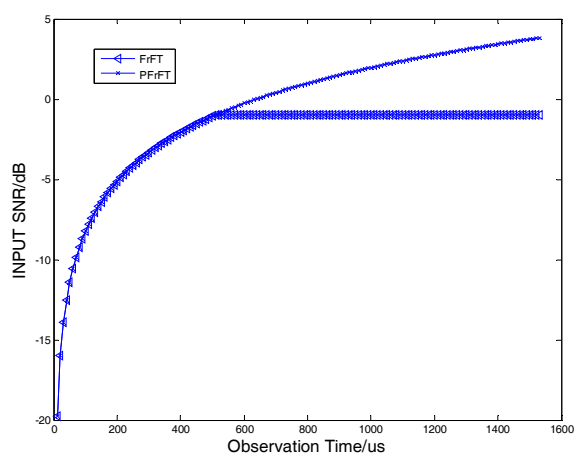

Figure 6. Compare PFRFT、FRFT output SNR

\subsection{Output SNR Analysis}

First-order perturbation method is uesed to analysis the PFrFT output SNR as follews. according to formula $(11), \delta P(\Omega)$ is interference term affecting the signals estimate, then

$$
P_{x}(\Omega)=P_{s}(\Omega)+\delta P_{s}(\Omega)
$$

Where

$$
\begin{aligned}
\delta P_{s}(\Omega) & =2 \operatorname{Re}\left[\operatorname{PFRFT}_{s}(\Omega) \cdot \operatorname{PFRFT}_{n}^{*}(\Omega)\right] \\
& =2 \operatorname{Re}\left[\int_{-\infty}^{+\infty} \int_{-\infty}^{+\infty} F_{\alpha}(\Omega) S_{1}\left(t_{1}\right) F_{\alpha}^{*}(\Omega) n^{*}\left(t_{2}\right) d t_{1} d t_{2}\right] \\
& =2 \operatorname{Re}\left[\frac{1-j \cot \alpha}{2 \pi} A \cdot e^{j u^{2} \cot \alpha} \int_{0}^{T_{0}} \int_{0}^{T_{0}} e^{j(2 \pi f-u \cdot \csc \alpha) \cdot t} \cdot e^{j \pi g \cdot \bmod \left(t_{1}, T_{z}\right)^{2}+\frac{\cot \alpha}{2} \bmod \left(t_{1}, \tilde{T}\right)^{2}} n^{*}\left(t_{2}\right) \cdot e^{-j \frac{\left(\bmod \left(t_{2}, \tilde{T}_{z}\right)\right)^{2}}{2} \cot \alpha+j u \cdot t_{2} \cdot \csc \alpha} d t_{1} d t_{2}\right]
\end{aligned}
$$

By the formula (18), due to the introduction of the noise signals, the peak coordinate of $P_{x}(\Omega)$ moves from the original place $\Omega_{0}=\left(f_{0}, g_{0}, T_{z}\right)$ to $\Omega_{0}+\delta \Omega_{0}=\left(f_{0}+\delta f_{0}, g_{0}+\delta g_{0}, T_{0}+\delta T_{z}\right)$, first-order partial derivatives of $P_{x}(\Omega)$ is 0 , with $\Omega_{0}+\delta \Omega_{0}$, there is

$$
\begin{aligned}
& \frac{\partial}{\partial f}\left[\left(P_{s}(\Omega)+\delta P_{s}(\Omega)\right]_{\substack{f_{0}+\delta f \\
g_{0}+\delta g}}=0\right. \\
& \frac{\partial}{\partial g}\left[\left(P_{s}(\Omega)+\delta P_{s}(\Omega)\right]_{\substack{g_{0}+\delta f \\
g_{0}+\delta g}}=0\right.
\end{aligned}
$$

With the Taylor formula at $\left(P_{s}(\Omega)+\delta P_{s}(\Omega)\right.$, the formula (19) (20) can be written as:

$$
\begin{gathered}
\frac{\partial}{\partial f}\left[P_{s}(\Omega)\right]_{f_{0}}+\frac{\partial}{\partial f}\left[\delta P_{s}(\Omega)\right]_{\substack{f_{0} \\
g_{0}}}+\frac{\partial^{2}}{\partial f^{2}}\left[P_{s}(\Omega)\right]_{\substack{f_{0} \\
g_{0}}} \cdot \delta f+\frac{\partial^{2}}{\partial g \partial f}\left[P_{s}(\Omega)\right]_{f_{0}} \delta g \cong 0 \\
\frac{\partial}{\partial g}\left[P_{s}(\Omega)\right]_{f_{g_{0}}}+\frac{\partial}{\partial g}\left[\delta P_{s}(\Omega)\right]_{f_{0}}+\frac{\partial^{2}}{\partial g^{2}}\left[P_{s}(\Omega)\right]_{\substack{g_{0} \\
g_{0}}} \cdot \delta g+\frac{\partial^{2}}{\partial g \partial f}\left[P_{s}(\Omega)\right]_{g_{0}} \delta f \cong 0
\end{gathered}
$$

Assuming

$$
B=\frac{\partial^{2}}{\partial f^{2}}\left[P_{s}(\Omega)\right]_{\substack{f_{0} \\ g_{0}}}=\frac{A^{2} T_{0}^{2} \csc \alpha_{0}}{12 \pi}
$$




$$
\begin{gathered}
C=\frac{\partial^{2}}{\partial g^{2}}\left[P_{s}(\Omega)\right]_{f_{0}}=\frac{A^{2} T_{0}^{6} \csc \alpha_{0}}{720 \pi} \\
u=\frac{\partial}{\partial f}\left[\delta P_{s}(\Omega)\right]_{f_{0}} \\
v=\frac{\partial}{\partial g}\left[\delta P_{s}(\Omega)\right]_{\substack{g_{0} \\
g_{0}}}
\end{gathered}
$$

$P_{s}(\Omega)$ gets extremism when $f=f_{0}, g=g_{0}$, then

$$
\begin{gathered}
\frac{\partial}{\partial f}\left[P_{s}(\Omega)\right]_{f_{0}}=0 \\
\frac{\partial}{\partial g}\left[P_{s}(\Omega)\right]_{f_{0}}=0 \\
\frac{\partial^{2}}{\partial f \partial g}\left[P_{s}(\Omega)\right]_{f_{g_{0}}}=0
\end{gathered}
$$

Therefore, formula (20) (21) can be expressed as:

$$
\left[\begin{array}{cc}
B & 0 \\
0 & C
\end{array}\right] \cdot\left[\begin{array}{c}
\delta f \\
\delta g
\end{array}\right]=\left[\begin{array}{l}
-u \\
-v
\end{array}\right]
$$

The estimation error of start frequency and MF rate can be expressed as:

$$
\begin{gathered}
\delta f=-\frac{u}{B} \\
\delta g=-\frac{v}{C}
\end{gathered}
$$

Formula (24) (25) show that $B, C$ are constants, $E(u)=0 、 E(v)=0$, so according to formula (32) (33), obtains

$$
\begin{gathered}
E(\delta f)=E\left(-\frac{u}{B}\right)=0 \\
E(\delta g)=E\left(-\frac{v}{C}\right)=0
\end{gathered}
$$

Therefore, the PFrFT multi-periods LFMCW signals parameters estimation is unbiased estimation.

$$
\begin{gathered}
\delta^{2} g=\frac{E\left[|u|^{2}\right]}{B^{2}}=\frac{6 \sigma_{0}^{2}}{A^{3} T_{0}^{3}}+\frac{6 \sigma_{0}^{4}}{A^{4} T_{0}^{4}} \\
\delta^{2} g=\frac{E\left[|u|^{2}\right]}{B^{2}}=\frac{360 \sigma_{0}^{2}}{A^{3} T_{0}^{5}}+\frac{360 \sigma_{0}^{4}}{A^{4} T_{0}^{6}}
\end{gathered}
$$

As can be seen in formula (36) (37), the estimating mean square error of start frequency and FM rate is decreases with observation time increasing, so, it is useful to improve the signals parameter estimation accuracy by increasing the observation time. It has been shown to be equivalent to the generalized likelihood ratio test (GLRT) and maximum likelihood estimator (MLE) in detection and estimation of LFMCW signals

\section{Conclusion}

This paper analyzes the advantages and disadvantages about LFMCW signals parameter estimation by FRFT, and proposes a multi-periods LFMCW signals parameter estimation algorithm basing PFrFT algorithm, the conclusions as follows: 
(1)When using FRFT to estimate multi-period LFMCW signals parameters, there are a plurality of peaks appearing in converting figure, and the SNR do not increase when the observation time is more than one period. It is means that FRFT is not optimal multi-period LFMCW signals parameter estimation algorithm.

(2) PFRFT output SNR is $\frac{S N R_{i n}^{2} \cdot T_{0}^{2}}{2 T_{0} \cdot S N R_{i n}+1}$, When the sample SNR is large enough, The PFRFT output SNR at the true parameter values varies approximately linearly with the signal observation time

(3) In the noise environment, estimating the multi-period LFMCW signals parameters with PFrFT is unbiased estimates.

(4) It is solved the cross-term problems of WVD, the resolution problems of SPWVD and TFDS, inadequate use of the sample data of FrFT.

\section{References}

Chen, P., Hou, C. H., \& Liang, Y. H. (2007). Research on Discrete FrFT-Based Detection Algorithm for LFM Echo of Underwater Moving Target. ACTA Armamentrii, 7(28), 834-838.

Deng, B., Tao R., Ping, D. F., \& Ma, L. (2009). Moving-Target-Detection Algorithm with Compensation for Doppler Migration Based on FRFT. ACTA Armamentrii, 10(30), 1304-1308.

Geroleo, F. G. (2012). Brandt-Pearce M. Detection and estimation of LFMCW radar signals. IEEE Transactions on Aerospace and Electronic Systems, 48(1), 405-418. http://dx.doi.org/10.1109/TAES.2012.6129644

Geroleo, F., G., \& Brandt, P. M. (2010). Detection and estimation of multi-Pulse LFMCW radar signals $[C] / /$ Radar Conference. Washington, DC: IEEE, 1009-1013.

Liu, F., Sun, D. P., \& Huang, Y. (2009). Fast parameter-estimation of LFM signals based on improved combined WVD and randomized hough transform. ACTA Armamentrii, 30(12), 1642-1646.

Liu, F., Xu, H., Tao, R., \& Wang, Y. (2012). Research on resolution among multi-component LFM signals in the fractional Fourier domain. Science China, 2(42), 136-148.

Liu, J. C., Liu, Z., \& Wang, X. S. (2007). SNR Analysis of LFMsignals with gaussian white noice in Fraictional Fourier Trandform domain. Journal of Electronics\& Information Technology, 10(29), 2338-2340.

Millioz, F., \& Davies, M. E. (2011). Detection and segmentation of FMCW radar signals based on the chirplet transform[C]//2011 IEEE International Conference on Acoustics, Speech and Signals Processing. Prague, CS: IEEE,:1765-1768.

Peter O'Shea. A. (2004). Fast Algorithm for Estimating the Parameters of a Quadratic FM Signals. Ieee Transactions on Signals Processing, 2(52), 385-393. http://dx.doi.org/10.1109/TSP.2003.821097

Qu, Q., \& Jin, M. L. (2009). Adaptive FrFT Based Chirp Signals Detection and Parameter Estimation. Journal of Electronics\& Information Technology, 12(31), 2937-2940.

Wang H. Y., Qiu, T. S., \& Chen, Z. (2008). Nonstationary Random Signals Analysis and Processing. Beijing:National Defense Industry Press, 311-313.

Wang, R., \& MA, Y. (2014). DOA Estimation of Wideband Linear Frequency Modulated Pulse Signals Based on FrFT. ACTA Armamentrii, 2(35), 207-213.

Wen, J. Y., Zhang, H., \& Wang, Y. (2012). Parameters estimation algorithm of LFM pulse compression radar signals. Transactions of Beijing Institute of Technology, 32(7), 746-750.

White, P. R., \& Locke, J. (2012). Performance of methods based on the FrFT for the detection of linear frequency modulated signals. IET Signals Processing, 6(5), 478-483. http://dx.doi.org/10.1049/iet-spr.2011.0189

\section{Copyrights}

Copyright for this article is retained by the author(s), with first publication rights granted to the journal.

This is an open-access article distributed under the terms and conditions of the Creative Commons Attribution license (http://creativecommons.org/licenses/by/3.0/). 